\title{
Factores bióticos y abióticos que condicionan la biorremediación por Pseudomonas en suelos contaminados por hidrocarburos
}

\author{
Sara Esmeralda Gómez Romero', Diana Carolina Gutiérrez Bustos', \\ Ana María Hernández Marín', Cindy Zulay Hernández Rodríguez', \\ Mariana Losada Casallas', Paula Cecilia Mantilla Vargas'?
}

1. Estudiantes de noveno semestre de Bacteriología y Laboratorio Clínico de la Universidad Colegio Mayor de Cundinamarca. 2008.

Correspondencia: sarita865@gmail.com

Recibido 25-04-08 / Aceptado 15-05-08

\begin{abstract}
Resumen
El proceso de biorremediación de hidrocarburos, contaminantes del suelo, llevado a cabo por Pseudomonas sp. está condicionado por factores bióticos y abióticos como los nutrientes, respiración, pH, humedad y temperatura propios del microorganismo, los cuales al ser modificados limitan o favorecen la capacidad metabólica de la bacteria y su adaptabilidad con el cambio de posición de los ácidos grasos de la membrana celular. La biorremediación de suelos ha demostrado ser una tecnología eficaz para el tratamiento de la contaminación por petróleo, debido a que gran parte de los componentes del crudo y sus productos refinados son biodegradables. La presente revisión tiene por objetivo describir las condiciones bióticas y abióticas que necesitan las Pseudomonas para llevar a cabo un adecuado proceso de biorremediación, teniendo en cuenta las características y propiedades de la bacteria.

Palabras clave: biodegradación, biorremedación, factores bióticos, factores abióticos, hidrocarburos, Pseudomonas.

\footnotetext{
Abstract

Biotic and abiotic factors that condition the bioremediation by Pseudomonas in grounds contaminated by hydrocarbons

The bioremediation of hydrocarbons process, polluting agents of the ground, carried out by Pseudomonas
} $s p$. is in conditioned by biotic and abiotic factors, like the nutrients, breathing, $\mathrm{pH}$, humidity and temperature of the microorganism, which, when modified, limit or favor the metabolic capacity of the bacterium and its adaptability with the shift of position of fatty acids of the cellular membrane. Ground bioremediation has demonstrated to be an effective technology for the treatment of the contamination by petroleum,
\end{abstract}


because the majority of the crude components and its refined products are biodegradable. This revision is to describe the biotic and abiotic conditions needed by the Pseudomonas sp. in order to carry out a suitable process of bioremediation, considering the characteristics and properties of the bacterium.

Key Words: abiotic factors, biodegradation, bioremediation, biotic factors, hydrocarbons, Pseudomonas.

\section{Introducción}

Debido al excesivo uso de combustibles y productos derivados del crudo de petróleo se ha incrementado la contaminación de los suelos por derrames de esta compleja mezcla de compuestos orgánicos, ocasionando un gran desequilibrio en el ecosistema que impacta de manera negativa al entorno ecológico $(1,2)$. Esto ha llevado a pensar en la biorremediación como una técnica efectiva en la descontaminación $(1,2)$ por medio de la degradación microbiana utilizando estrategias como la bioestimulación de microorganismos nativos, y el bioaumento (3-5), basado en la inoculación de cepas o consorcios microbianos de laboratorio $(6,7)$.

En los ambientes contaminados existen en abundancia especies con estas capacidades metabólicas específicas, debido a la adaptación de la microflora presente en el contaminante (8). Las Pseudomonas spp son una opción muy viable., ya que son microorganismos capaces de utilizar estos hidrocarburos como fuente de carbono y energía (9) necesaria para su crecimiento, generando productos más sencillos. Para que estos procesos metabólicos se lleven a cabo y puedan ser utilizados como una técnica de biorremediación, es necesario conocer las condiciones físicas químicas y de nutrición adecuadas del medio $(10,11,12)$.

En cuanto a las condiciones físicas y químicas, existen varios factores que condicionan la biorremediación de un suelo; entre las físicas se encuentran factores ambientales como el pH (2), la temperatura (3), concentración de minerales, humedad del suelo, respiración y nutrientes disponibles. Entre las químicas encontramos la estructura molecular del contaminante, su concentración y la presencia de una población microbiana potencialmente activa (13).

\section{Factores bióticos}

\section{Suelo}

El suelo está formado por una cubierta superficial llamada corteza terrestre o litosfera, que es la capa más delgada de las que forman la tierra y constituye un conjunto complejo de elementos físicos, químicos y biológicos. Uno de esos componentes son los minerales, que se forman por la meteorización de las rocas, de materia orgánica, como el humus y la biomasa viva y muerta, de gas y de agua. Todos ellos desempeñan funciones naturales como la descomposición de productos vegetales o la biodegradación de ciertos componentes que lo contaminan por medio de procesos metabólicos que llevan a cabo los microorganismos $(14,15,16)$.

La estructura del suelo se encuentra dividida en tres tipos de capas a las que se les llama horizontes y su diferenciación se debe tanto a la dinámica interna, ya sea por su composición y textura, como al transporte vertical debido a la lixiviación o la capilaridad. El horizonte A es el más superficial, en el cual abunda materia orgánica descompuesta o humus, el contacto de este con las bacterias facilita la degradación de hidrocarburos como el fenantreno (17). Esta zona se puede ver alterada por incendios, explotación de minas y contaminación por componentes que no se degradan. El horizonte B, también llamado subsuelo, es el más grande, se compone de materiales provenientes de fragmentos de rocas como la arena gruesa y liviana, que cumple funciones de filtración; el limo, el cual mantiene la homeostasis y la arcilla, que retiene el agua. El horizonte $\mathrm{C}$ está compuesto de material rocoso que no ha sufrido ninguna alteración química o física (15). 
Los contaminantes del petróleo se alojan principalmente en el horizonte $A$, donde se encuentra el mayor contenido de materia orgánica que incluye a los microorganismos $(18,19)$ que pueden ser estimulados por la adición de nutrientes, ya sean fertilizantes (20), o de Oxígeno por medio de la agitación. Existen otros tipos de contaminantes como los hidrofóbicos (21) que se absorben y se depositan en los poros del suelo, dificultando la biodegradación (3).

Los suelos expuestos a la contaminación prolongada con hidrocarburos derivados del petróleo, constituye un microhábitat adecuado para la evolución de especies bacterianas degradadotas. El contaminante ejerce una presión sobre la población microbiana involucrada y de esta forma se seleccionan aquellos capaces de sobrevivir y adaptarse a estas condiciones (22-25).

\section{Capacidad metabólica del microorganismo}

En el proceso de biorremediación la degradación efectuada por la Pseudomonas spp, se debe tener en cuenta que al ser parte de una población nativa, está interactuando con otros microorganismos formando los llamados consorcios microbianos. En este se establecen procesos simbióticos $(26,27,28)$ como el de Arthrobacter y Pseudomonas spp para la degradación del fluoreno, sin la acumulación de 9-fluorenona, metabolito de oxidación intermedia (29), o de comensalismo con un cultivo mixto de Rhodococcus erythopolis, Bacillus cereus y Pseudomonas fluorescens para biodegradar hidrocarburos poliaromáticos. Esta interrelación, que se fundamenta en que el cultivo mixto, crece a expensas de los contaminantes, mientras que P. aeruginosa GL1 degrada los metabolitos por la ruptura en la posición meta, estabilizando la población microbiana en el cultivo (30). Relaciones y condiciones naturales que han sido muy difíciles de recrear en el laboratorio.

Con referencia a la biomasa, se debe tener en cuenta la cinética de crecimiento del microorganismo obteniendo mayor rendimiento metabólico en la fase exponencial, debido a que el aumento de esta es un indicador del proceso de biorremediación. $(3,24,31)$. Se ha logrado estimar que la cantidad suficiente de microorganismos para efectuar en buenas condiciones un proceso de biodegradación es de 103 a 104 UFC/g suelo y de heterótrofos totales de 105 a 106 UFC/g de suelo (32), capaces de metabolizar y mineralizar el contaminante a $\mathrm{CO}_{2}$ y $\mathrm{H}_{2} \mathrm{O}(33,34)$, compuestos que permiten efectuar el reciclaje en el medio ambiente al ser fácilmente incorporados en los ciclos biogeoquímicos.

La conformación genética de Pseudomonas es muy versátil; por poseer operones, elementos móviles como transposones y plásmidos (35), que permiten la transferencia de los genes $(31,27)$ y, por lo tanto, la rápida adaptación frente a la presencia de agentes contaminantes nuevos en un ecosistema en particular $(27,36)$. Además, poseen genes que codifican para enzimas, con las que se lleva a cabo la mineralización del contaminante $(2,19)$. Se ha estudiado la producción en la fase estacionaria de biosurfactantes o tensoactivos por parte de la Pseudomonas aeruginosa cuyo papel en la biorremediación es de solubilizar los compuestos hidrofóbicos (37-40).

En los derrames de petróleo la mayoría de compuestos alifáticos se pierden por volatilización, mientras que los hidrocarburos policíclicos permanecen, generando un gran impacto en virtud de los efectos recalcitrantes y tóxicos parar los seres vivos (41). Los hidrocarburos poliaromáticos (HAPs) más estudiados son los monoaromáticos BTEX, el diaromático naftaleno y los triaromáticos fenantreno, antraceno y fluoreno (42), que generan un impacto negativo a los ecosistemas $y$ afecta la salud de los seres vivos (43).

El proceso de biorremediación se inicia con la oxidación del anillo aromático mediante la incorporación de dos átomos de oxígeno catalizado por una dioxigenasa (44). A partir de esta reacción se forma un cis-dihidrodiol y el anillo pierde la aromaticidad. A continuación una deshidrogenasa NAD+ dependiente, reconstituye el anillo aromático formando un catecol (diol). Los dioles son moléculas a partir de las cuales se produce la ruptura del anillo aromático mediante dioxigenasas estereoselectivas (la ruptura se da entre los dos grupos hidroxilo adyacente, denominándose meta-ruptura) (45-47).

Sin embargo, este proceso puede ser limitado por la estructura química del contaminante, ya que al incrementar la cantidad de enlaces y grupos funcionales $(26,48)$ o al polimeralizarse resulta más difícil la incorporación al metabolismo bacteriano, especialmente cuando presentan grupos halógenos (49) y metales pesados, catalogados como tóxicos para los microorganismos $(3,50)$. 


\section{Nutrientes}

Los nutrientes son uno de los factores más relevantes por ser sustancias químicas necesarias para la actividad microbiana y metabólica de la Pseudomonas spp, por lo que estos constituyentes se deben encontrar disponibles para su asimilación y síntesis, y deben ser controlados para aumentar la eficiencia y el buen desarrollo de la biorremediación. Se dividen en dos grandes grupos: macronutrientes y micronutrientes $(14,51)$.

Los macronutrientes de mayor importancia metabólica se encuentran; el carbono (C) en este caso los hidrocarburos como contaminantes proporcionan la energía necesaria para la fabricación de compuestos celulares y productos metabólicos (dióxido de carbono, agua, enzimas) (6); el Nitrógeno $(\mathrm{N})$, elemento necesario para la producción de aminoácidos, proteínas, enzimas, ácidos nucleicos y otros constituyentes celulares. En ocasiones la utilización de estos nutrientes es rápida, los suelos no alcanzan a cubrir todas las necesidades, siendo un factor limitante para la degradación microbiana, por lo cual se incorpora fertilizantes de uso agrícola como urea o sulfato de amonio y de origen orgánico como estiércol, para acelerar el proceso de biorremediación $(52,53)$.

El fósforo (P) que interviene en la formación de compuestos energéticos dentro de las células y es requerido para la síntesis de ácidos nucleicos y fosfolípidos en los procesos de reproducción y degradación, puede ser adicionado al suelo como fosfato diamónico o fosfato tricálcico. El Potasio $(\mathrm{K})$ requerido por una gran cantidad de enzimas para catalizar diferentes reacciones (10).

Por lo general suele haber en el suelo una concentración de macronutrientes suficiente, sin embargo, sí estos no se encuentran en el rango normal se puede adicionar mayor cantidad al medio. Si la concentración de nutrientes inorgánicos como $\mathrm{N}$ y $\mathrm{P}$ es baja, se produce una relación $\mathrm{C} / \mathrm{N}$ y $\mathrm{C} / \mathrm{P}$ muy altas, lo no es favorable para el crecimiento microbiano, de acuerdo a la relación C:N:P dentro de un rango normal de 100:10:1 a 100:2:0,2 (54).

La fuente de micronutrientes, oligoelementos o elementos minoritarios constituye un conjunto variado de elementos entre los que se encuentra el hierro $(\mathrm{Fe})$, cobre $(\mathrm{Cu})$, zinc $(\mathrm{Zn})$, azufre $(\mathrm{S})$, cobalto (Co), manganeso $(\mathrm{Mn})$, magnesio $(\mathrm{Mg})$ y calcio (Ca), normalmente no se incorporan en el proceso de biorremediación, puesto que el suelo provee estos elementos en cantidades suficientes $(55,56)$.

\section{Respiración y aireación}

La biorremediación es fundamentalmente un "proceso metabólico de transferencia de electrones". La energía necesaria para el crecimiento microbiano se obtiene durante el proceso de oxidación de materiales reducidos, donde las enzimas microbianas catalizan la transferencia de los electrones (57). Este proceso se denomina "respiración microbiana”, y se basa en que en la cadena respiratoria, o transportadora de electrones de las células, producen una serie de reacciones de óxido-reducción cuyo fin es la obtención de energía. La cadena la inicia un sustrato orgánico (compuestos hidrocarburados) que es externo a la célula y actúa como dador de electrones, de modo que la actividad metabólica de la célula acaba degradando y consumiendo dicha sustancia. Los aceptores más utilizados por los microorganismos en general y particularmente por las Pseudomonas spp, son el oxígeno y los nitratos $(14,57,58)$.

Cuando el oxígeno es utilizado como aceptor de electrones la respiración microbiana se produce en condiciones aerobias, los microorganismos convierten en última instancia los contaminantes en dióxido de carbono, agua y masa celular microbiana de las Pseudomonas (mineralización) por enzimas oxigenasas (3). Sin embargo, sí utiliza los sulfatos o el dióxido de carbono, se produce en condiciones reductoras o anaerobias $(14,33,34,59)$.

El proceso de biorremediación aerobia y anaerobia puede esquematizarse de la siguiente manera (10):

Degradación aerobia:

Sustrato $+\mathrm{O}_{2}=$ biomasa $+\mathrm{CO}_{2}+\mathrm{H}_{2} \mathrm{O}$

Degradación anaerobia:

Sustrato + $\left(\mathrm{NO}_{3}^{-}, \mathrm{SO}_{4}^{-}, \mathrm{Fe}^{3+}, \mathrm{Mn} 4+, \mathrm{CO}_{2}\right)=$ Biomasa $+\mathrm{CO}_{2}+\left(\mathrm{N}_{2}, \mathrm{Mn}^{2+}, \mathrm{S}^{2+}, \mathrm{Fe}^{2+}, \mathrm{CH}_{4}\right)$

El oxígeno generalmente es el mejor aceptor de electrones, es decir, el que produce la mayor energía libre en una reacción completa. En consecuencia, para un mismo substrato orgánico, los microorganismos que emplean el oxígeno como agente oxidante pueden generar mayor energía que aquellos que emplean nitratos, sulfatos ú otros aceptores de electrones alternativos, 
logrando de esta forma crecer a mayor velocidad, lo que implica un mayor consumo del sustrato. Por lo tanto la biorremediación aerobia es típicamente más eficiente que la biorremediación de contaminantes orgánicos en forma anaerobia (60).

Para cuantificar el proceso se utiliza la medición del $\mathrm{CO}_{2}$ producido por unidad de tiempo en un área determinada. Esta es una medida indirecta del proceso biodegradativo, cuyo objetivo es evaluar a través de métodos como el de respirometría, la actividad metabólica de los microorganismos del suelo durante el proceso de degradación de los compuestos orgánicos $(14,61,62)$.

El flujo de oxígeno es vital para la viabilidad del microorganismo. Se estimula drenando y/ó volteando el suelo no saturado, para incorporar el oxígeno atmosférico en el sistema (63). Entre otras técnicas encontramos la biorrecuperación, que consiste en la ventilación forzada, mediante la inyección a presión de oxígeno en la zona no saturada del suelo $(63,64)$.

Debido a la aireación del suelo se favorece la degradación de los hidrocarburos por dos motivos: por volatilización, facilitando la migración de la fase volátil de los contaminantes, y por biodegradación, ya que al incrementar la oxigenación del suelo se va a estimular la actividad bacteriana (10). Se tienen en cuenta los siguientes factores: se degradarán más fácilmente las moléculas más pequeñas (hasta $\mathrm{CO}_{2}$ ), siendo más fácilmente biodegradables los compuestos parafinados o de cadena lineal que los compuestos aromáticos.

En general, son favorables los compuestos de alta volatilidad (presión de vapor mayor de $10 \mathrm{~mm}$ de $\mathrm{Hg}$ a $20^{\circ} \mathrm{C}$ ). Los suelos deben contener bajos contenidos en arcilla y ser homogéneos, con un valor de permeabilidad al aire adecuado $\left(>1010 \mathrm{~cm}^{2}\right)$. Cuanto menor es la solubilidad de los contaminantes menor será la biodisponibilidad de los microorganismos. Los aportes de oxígeno deben ser suficientes, así como la existencia de fuentes de carbono, aceptores de electrones y energía que complementan el proceso $(10,14)$. El residuo debe ser susceptible de biodegradarse biológicamente, y presentarse en una forma física a los microorganismos, es decir, que el producto obtenido de la biodegradación no vaya a ser más tóxico que el contaminante $(3,50)$.

\section{Factores abióticos}

\section{$\mathrm{pH}$}

$\mathrm{El} \mathrm{pH}$ es un factor químico importante que influye en la recuperación de suelos contaminados por hidrocarburos, ya que puede afectar principalmente a las poblaciones de Pseudomonas y la biodisponibilidad de las fuentes de carbono y energía. Este factor se constituye como uno de los indicadores del proceso de biorremediación y aunque las Pseudomonas se pueden adaptar fácilmente a condiciones extremas, estas cepas microbianas tienen un determinado rango de tolerancia $(50,65)$.

A pH extremadamente alcalinos o extremadamente ácidos la biodegradación se hace lenta (66). Generalmente los suelos contaminados por hidrocarburos tienden a ser ácidos, lo cual limita el crecimiento y la actividad de las Pseudomonas El rango óptimo para la biodegradación está entre 6-8 pH (33). Sin embargo, para mantener una mejor capacidad degradante, por periodos de tiempo prolongados, el $\mathrm{pH}$ debe ser neutro, entre 7.4-7.8, evitando al máximo las fluctuaciones $(3,50)$.

Es necesario tener en cuenta que la variación del $\mathrm{pH}$, además de afectar la actividad microbiana, también afecta la solubilización, adsorción y absorción de los contaminantes y de los iones (67). Las formas catiónicas $\left(\mathrm{NH}^{4+}, \mathrm{Mg} 2+, \mathrm{Ca}^{2+}\right)$ son más solubles a $\mathrm{pH}$ ácido mientras que las formas aniónicas $\left(\mathrm{NO}_{3}{ }^{-}, \mathrm{NO}_{2}{ }^{-}, \mathrm{PO}_{4}{ }^{3-}, \mathrm{Cl}^{-}\right)$son más solubles a pH alcalino (68-70). Por lo tanto, si es necesario alcalinizar un suelo se utiliza arena de caliza e iones $\mathrm{Ca}^{2+} \mathrm{y} \mathrm{Mg}^{2+}$, mientras que para acidificar un suelo se utiliza $\mathrm{FeSO}_{4}$ (71). En otros estudios se ha reportando que la acidificación o la reducción del $\mathrm{pH}$ en el suelo se puede realizar adicionando compuestos del azufre (72).

\section{Humedad y temperatura}

Las Pseudomonas spp son bacterias que requieren unas condiciones mínimas de humedad para su crecimiento; el agua es importante para su desarrollo porque actúa como medio de transporte de nutrientes y oxígeno a la célula ya que forma parte de su protoplasma bacteriano. Es conveniente mantener una humedad del orden del 25 - $75 \%$ de la capacidad de campo, la cual se define como la masa de agua que admite el suelo hasta la saturación, que depende de cada tipo de suelo (73). 
La biodegradación de hidrocarburos en ecosistemas terrestres puede ser limitada por la cantidad de agua disponible en el medio para el crecimiento y metabolismo de las Pseudomonas. Diferentes estudios reportan que las tasas óptimas de biodegradación se dan cuando la saturación de agua en el suelo se encuentra entre el 30\% a 90\%. En valores menores la degradación se ve inhibida (14).

La humedad del sitio a tratar es un factor importante para la elección de una tecnología en particular. Una alta humedad puede impedir la transferencia de gases a través del suelo, que afecta los procesos de biorremediación, así como problemas durante la excavación y transporte, además de aumentar costos durante el uso de métodos de remediación térmicos (74). Una baja humedad reduce la actividad metabólica bacteriana (50). Al igual que la humedad, la temperatura es un factor ambiental que se debe tener en cuenta en el momento de la aplicación de la técnica de biorremediación así como en la evaluación de la eficacia del procedimiento (13).

La biorremediación llevada a cabo entre $20^{\circ} \mathrm{C} \mathrm{y} 40^{\circ} \mathrm{C}$ muestra que este intervalo de temperatura es óptimo para la actividad microbiana $(29,45)$, sin embargo en climas tropicales es mejor una temperatura aproximadamente de 30 a $35^{\circ} \mathrm{C}$ (32) para la actividad de las Pseudomonas. La velocidad de degradación aumenta con la temperatura, por lo que un incremento de la misma es útil. Cuando la temperatura se incrementa en $10^{\circ} \mathrm{C}$ la biorremediación se duplica, pero se elevan los costos. Para mantener constante este factor se hace necesario cubrir la zona del derrame ya sea con paja, vegetación o plástico para conservar la radiación solar, o la utilización de electrodos enterrados en el suelo o la circulación constante de agua caliente (19).

Cuando se supera los $40^{\circ} \mathrm{C}$ se produce una disminución de la actividad microbiana, una rotación poblacional hacia especies más resistentes a las altas temperaturas (32) o puede decrecer la biorremediación debido a la desnaturalización de enzimas y proteínas de las bacterias. Cuando la temperatura esta a $0^{\circ} \mathrm{C}$ se detiene esencialmente la biodegradación (45). Cuando ocurren derrames de petróleo en regiones cuyas temperaturas son entre $1 \mathrm{a} 15^{\circ} \mathrm{C}$ se suelen usar microorganismos psicrófilos (75).

Frente a los cambios de humedad y temperatura las Pseudomonas presentan transformaciones estructurales que permiten su adaptación al medio. La deshidratación de la membrana a temperatura constante causa una transformación de fases en ella, que produce un aumento en la temperatura de transición. Una deshidratación extensa puede causar un cambio en la bicapa lipídica de la membrana, formando micelas entre los fosfolípidos (76). Las Pseudomonas spp. que se ven expuestas a bajos potenciales hídricos y por ende a altas temperaturas, ajustan la composición de los ácidos grasos de su membrana o efectúan otras adaptaciones para minimizar los efectos de solidificación de los lípidos por consecuencias de la deshidratación.

Las Pseudomonas spp. responden a cambios ambientales modificando su composición de ácidos grasos de la membrana celular que le permite mantener la homeoviscosidad ante situaciones de estrés y su posterior adaptabilidad. Además, los cambios de temperatura las Pseudomonas spp. presentan un incremento de la insaturación de ácidos grasos con la disminución de esta con el fin de mantener la fluidez de la membrana, y ante el aumento de temperatura hay un incremento de ácidos grasos saturados y una disminución de los ácidos grasos insaturados (12), demostrando de esta manera que las Pseudomonas spp. son microorganismos euritérmicos, es decir, microorganismos capaces de adaptarse a los cambios, lo cual hace que estas bacterias sean las mejores aliadas en la aplicación de la biorremediación de suelos contaminados con hidrocarburos en diferentes condiciones ambientales de temperatura.

Teniendo en cuenta la revisión realizada se puede anotar que en los ambientes contaminados con hidrocarburos derivados del petróleo, se ejerce una selección natural de aquellas especies con capacidad adaptativa para crecer en presencia de estos compuestos, microorganismos ideales para los procesos de biorremediación.

La información genética referente a la capacidad metabólica del microorganismo, es un factor que limita el proceso de biorremediación, ya que si no se tiene la codificación genética para poder realizar el catabolismo por medio de la acción enzimática, el microorganismo no es capaz de asimilar el contaminante como fuente de energía y nutrientes. Caso contrario es lo que ocurre con las Pseudomonas, que al poseer enzimas como las dioxigenasas (45-47) y deshidrogenasa (44), son capaces 
de degradar los hidrocarburos poliaromáticos, uno de los principales componentes del petróleo.

Para finalizar, es necesario anotar que para favorecer la actividad microbiana y metabólica los nutrientes juegan un papel esencial por lo cual se deben encontrar en las cantidades apropiadas para su asimilación y síntesis según su requerimiento. La biorremediación llevada a cabo entre 20 y $40^{\circ} \mathrm{C}(29,45)$ demuestra que este intervalo de temperatura es óptimo para la actividad de las Pseudomonas Los cambios de humedad al igual que los de temperatura llevan a que las Peudomonas presenten cambios estructurales que permitan su adaptación al medio (76). Es imperioso mantener el flujo de oxígeno para favorecer la respiración de los microorganismos, para ello, se hace uso de técnicas que ayuden en el proceso de respiración para la biorremediación.

$\mathrm{El} \mathrm{pH}$ siendo un factor abiótico importante para la biorrecuperación de suelos contaminados por hidrocarburos (50), puede ser modificado con arena caliza para alcalinizarlo o $\mathrm{FeSO}_{4}$ (71) y azufre para acidificarlo. Es necesario tener en cuenta que la biorrecuperación de suelos incluye factores bióticos y abióticos para ser exitosa y por lo tanto este trabajo debe ser interdisciplinario: Ingenieros para la aireación, geólogos, biólogos y agrónomos para el estudio de suelos y Bacteriólogos y Microbiólogos para la biorremediación con microorganismos.

\section{Agradecimientos}

A las docentes Sonia Marcela Rosas, Lucia Constanza Corrales y Ligia Consuelo Sánchez de la Universidad Colegio Mayor de Cundinamarca, por sus aportes en la elaboración de este artículo. NOV

\section{Referencias}

1. López JB, Quintero G, Guevara AL, Jaimes DC, Gutiérrez SM, García J. Bioremediación de suelos contaminados con hidrocarburos derivados del petróleo. Nova. 2006;4:82-90.

2. Ferrera R, Rojas N, Poggi HM, Alarcón A, Cañizares RO. Processes of bioremediation of soil and water which were contaminated by oil hydrocarbons and other organic substances. Rev Latinoam Microbiol. 2006; 48:179-187.

3. Leahy JG, Colwell RR. Microbial Degradation of Hydrocarbons in the Environment Microbiol Mol Biol Rev. 1990;54:305-315.

4. Ivshina IB, Kuyukina MS, Philp JC, Christofi N. Oil desorption from mineral and organic materials using biosurfactant complexes produced by Rhodococcus sppecies. World J. Microbiol. Biotechnol. 1998;14:711-717.
5. Mishra S, Jyot J, Kuhad RC, Lal B. Evaluation of inoculum addition to stimulate in situ bioremediation of oily-sludge-contaminated soil. Appl Environ Microbiol. 2001;67:1675-1681.

6. Mc Dougald D, Rice S, Weichart D, Kjelleberg S. Nonculturability: adaptation or debilitation? FEMS Microbiol Ecol. 1998;25:1-9.

7. Van Hamme JD, Singh A, Ward OP. Recent Advances in Petroleum Microbiology. Microbiol Mol Biol Rev. 2003;67:503-549.

8. Marchal R, Pene S, Solano-Serena F, Vandecasteele JP. Gasoline and Diesel Oil Biodegradation. Oil \& Gas Science and Technology. 2003;58: 441-448.

9. Bracho M, Díaz L, Soto L. M. Biodegradación de hidrocarburos aromáticos policíclicos y heterocíclicos por Pseudomonas spp. Ciencia. 2004;12:269-275.

10. Maroto ME, Rogel JM. Aplicación de sistemas de biorremediación de suelos y Aguas contaminadas por hidrocarburos. MMWR 2002. URL disponible en: http://aguas.igme.es/igme/publica/ pdflib15/028.pdf.

11. Atlas RM. Petroleum microbiology. New York: Macmillan Publishing Co; 1984.

12. Wilkinson S. G. Gram-negative bacteria. London: Academic Press; 1998.

13. Atlas RM. Microbial degradation of petroleum hydrocarbons: an environmental perspective. Microbiol Rev. 1981; 45(1): 180-209.

14. Viñas Canals Marc. Biorremediación de suelos contaminados por hidrocarburos: Caracterización microbiológica, química y ecotoxicológica. [Tesis Doctoral]. Barcelona: Universidad de Barcelona; 2005

15. Llorente Miguel Isidro. Resumen del Manual de Edafología Disponible en: http://www.usal.es/-delcien/doc/FS.PDF. Consultado: 27 de Febrero de 2008.

16. Foster L, Nannipieri P, Oades JM. Soil structure and biological activity. Disponible en: http://www.cababstractspplus.org/google/ abstract.aspp?AcNo=19971903099.html.

17. Ortega Calvo JJ, Jiménez CS. Effect of humic fractions and clay on biodegradation of phenanthrene by a Pseudomonas fluorescens strain isolated from soil. Appl Environ Microbiol. 1998;64:3123-3126.

18. Paul EA, Clark FE. Soil microbiology and biochemistry. San Diego: Academic Press; 1998.

19. Bossert I, Bartha R. The fate of petroleum in soil ecosystems. In: R.M. Atlas Ed. Petroleum microbiology. New York: Macmillan Publishing Co; 1984. pg. 434-476.

20. Atlas R M, Bartha R. Stimulated biodegradation of oil slicks using oleophilic fertilizers. Environ Sci Technol 1973;7:538-541.

21. Mihelcic JR, Lueking DR, Mitzell RJ, Stapleton JM. Bioavailability of sorbed-and separate-phase chemicals. Biodegradation.1993;4:141-153.

22. Schwarz JR, Walker JD, Colwell RR. Deep-sea bacteria: growth and utilization of n-hexadecane at in situ temperature and pressure. Can J Microbiol. 1975; 21:682-687.

23. Jensen V. Bacterial flora of soil after application of oily waste. Oikos. 1975; 26:152-158.

24. Pinholt Y, Struwe S, Kjoller A. Microbial changes during oil decomposition in soil. Ecography. 2006; 2:195-200.

25. Lianos C, Kjoller A. Changes in the flora of soil fungi following oil waste application. Oikos. 1976;27:377-382. 
26. Rojas J, Moreno S, Rondón M. Biodegradación de gasoil rango Diesel por un cultivo mixto de bacterias. Ciencia. 2005;13:23-35.

27. Sussman M, Collins CH, Skinner FA. Stewart DE. Release of genetically-engineered microorganisms. London: Academic Press; 1988.

28. Atlas R. M., Busdosh. M. Microbial degradation of petroleum in the Arctic. En: Proceedings of the 3rd International Biodegradation Symposium. London: Applied Science Publishers Ltda; 1976. p. 79-86.

29. Casellas M., Grifoll, M., Sabate J., Solanas A. M. Isolation and characterization of a 9- fluorenone-degrading bacterial strain and its role in synergistic degradation of fluorene by a consortium. Can J Microbiol. 1998; 44:734-742.

30. Pérez S. Biodegradación del naftaleno por Pseudomona aeruginosa. AT18. Tecnología Química 2003; 23:21-27.

31. Sayler GS, Shields MS, Tedford ET, Breen A, Hooper SW, Sirotkin KM, Davis JW. Application of DNA-DNA colony hybridization to the detection of catabolic genotypes in environmental samples. Appl Environ Microbiol. 1985;49:1295-1303.

32. Ercoli E, Gálvez J, Di Paola M, Cantero J, Videla S, Medaura M, Bauzá J. Análisis y evaluación de parámetros críticos en biodegradación de hidrocarburos en suelo. Disponible en: http:// www.eduardoercoli.com.ar/publicaciones/Param_criticos.pdf.

33. Alexander M. Biodegradation and bioremediation. San Diego: Academic Press; 1994.

34. Knaebel DB, Federle TW, McAvoy DC. Vestal JR. Effect of mineral and organic soil constituents on microbial mineralization of organic compounds in a natural soil. Appl Environ Microbiol. 1994;60:4500-4508.

35. Chakrabarty AM. Plasmids in Pseudomonas. Annu Rev Genet. 1976;10:7-30.

36. Van JR, Vos WM, Harayama S, Zehnder AJ. Molecular mechanisms of genetic adaptation to xenobiotic compounds. Microbiol Rev. 1992;56:677-694.

37. Nelson KE, Weinel C, Paulsen IT, Dodson RJ, Hilbert H, Martins VA, Fouts DE, y col. Complete genome sequence and comparative analysis of the metabolically versatile Pseudomonas putida KT2440. Environ Microbiol. 2002; 4:799-808.

38. Demanèche S, Kay E, Gourbière F, Simonet P. Natural transformation of Pseudomonas fluorescens and Agrobacterium tumefaciens in soil. Appl Environ Microbiol. 2001; 67:2617-2621.

39. Deziel E, Paquette G, Villemur R, Lepine F, Bisaillon J. Biosurfactant Production by a Soil Pseudomonas Strain Growing on Polycyclic Aromatic Hydrocarbons. Applied and environmental microbiology.1996;62:1908-1912.

40. Hommel RK. Formation and physiological role of biosurfactants produced by hydrocarbon-utilizing microorganisms. Biodegradation. 1990;1:107-119.

41. Tissot B, Welte DH. Petroleum formation and occurrence. New York USA: Sppringer-Verlang; 1984.

42. Kästner M, Mahro B. Microbial degradation of polycyclic aromatic hydrocarbons in soils affected by the organic matrix of compost. Appl Microbiol Biotechnol.1996;44:668-675.

43. White K. L. An overview of immunotoxicology and carcinogenic polycyclic aromatic hydrocarbons. Environ Carcinogen Rev. 1986;4:163-202.
44. Daane LL, Harjono I, Zylstra GJ, Häggblom MM. Isolation and characterization of polycyclic aromatic hydrocarbon-degrading bacteria associated with the rhizospphere of salt marsh plants. Appl Environ Microbiol. 2001;67:2683-2691.

45. Volke T, Velasco J. Biodegradación de hidrocarburos del petróleo en suelos intemperizados mediante composteo. Disponible en: http:// www.ine.gob.mx/dgcenica/descargas/composteo2003.pdf.

46. Adam R, Domínguez V, García L. Potencial de la Biorremediación de suelo y agua impactados por petróleo en el trópico Mexicano. Disponible en: www.chapingo.mx/terra/ contenido/17/2/art159-174.pdf.

47. Zhongqi H, Sppain J. Studies of the catabolic pathway of degradation of nitrobenzene by Pseudomonas pseudoalcaligenes JS45: Removal of the Amino Group from 2-Aminomuconic Semialdehyde. Appl Environ Microbiol. 1997;63:4839-4843.

48. Walker JD, Petrakis L, Colwell RR. Comparison of the biodegradability of crude and fuel oils. Can J Microbiol. 1976;22:598-602.

49. Suflita JM, Horowitz A, Shelton DR, Tiedje JM. Dehalogenation: a novel pathway for the anaerobic biodegradation of haloaromatic compounds. Science. 1982;218:1115-1117.

50. Dibble JT, Bartha R. Effect of environmental parameters on the biodegradation of oil sludge. Appl Environ Microbiol. 1979;37:729-739.

51. Swindoll C, Michael C, Aelion M, Pfaender F. Influence of inorganic and organic nutrients on aerobic biodegradation and on the adaptation respponse of subsurface microbial communities. Appl Environ Microbiol. 1988;54:212-217.

52. Cantwell SG, Lau EP, Watt DS, Fall RR. Biodegradation of acyclic isoprenoids by Pseudomonas species. J Bacteriol. 1978; 135:324-333.

53. Pardo JL, Perdomo MC, Benavides JL. Efecto de la adición de fertilizantes inorgánicos compuestos en la degradación de hidrocarburos en suelos contaminados con petróleo. NOVA. 2004; $2: 40-49$

54. Krieg NR, Holt JG. Bergey's Manual of Sistematic Bacteriology. Ed. Williams y Wilkings. Baltimor, U.S.A. 1984;1:141-199.

55. Pineda G, Mesta AM. Petroleum aspphaltenes: generated problematic and possible biodegradation mechanisms. Rev Latinoam Microbiol. 2001;43: 143-150.

56. Pereira JN, Morgan ME. Nutrition and physiology of Pseudomonas fragi. J Bacteriol. 1957;74:710-713.

57. Pellini A. Biorremediación estimulada por efluentes cloacales tratados de suelos contaminados con hidrocarburos. Disponible en: http://www.tesis.bioetica.org/lp.htm.

58. González R, Monroy M, Mier MV. Estudio de biorremediación de suelos contaminados con Tetracloroetileno. En: XXXVI Congreso Mexicano de Química. México Universidad Autónoma Metropolitana-Azcapotzalco: Sociedad Química de México, A.C; 2001. pg. 225-230.

59. Perry JJ. Microbial cooxidations involving hydrocarbons. Microbiol Rev 1979;43:59-72.

60. Eweis JB, Ergas SJ, Chang DP, Schroeder ED. Principios de Biorrecuperación: Tratamientos para la descontaminación y regeneración de suelos y aguas subterráneas mediante procesos biológicos y fisicoquímicos. Madrid: 1999. 
61. Adams RH, Armenta G, García L. Bioremediation in the petroleum producing region of southeast Mexico: En: Second Inter-American Environmental Congress. México: Instituto Tecnológico y de Estudios Superiores de Monterrey (ITESM); 1995.

62. Adams R, Domínguez V, Garciá L. Bioremediation Potential of Oil Impacted Soil and Water in the Mexican Tropics. Terra Latin. 1999; 17: 25-28.

63. Kincannon, CB. Oily waste dispposal by soil cultivation process. Dispponible en: http://www.amazon.com/dispposal-cultivationEnvironmental-protection-technology.html.

64. EPA. Bioremediation of Hazardous Waste Sites: Dispponible en: http://www.epa.gov/nrmrl/pubs/625k96001/625k96001.pdf.

65. Hambrick GA, DeLaune RD, Patrick WH. Effect of estuarine sediment $\mathrm{pH}$ and oxidation-reduction potential on microbial hydrocarbon degradation. Appl Environ Microbiol. 1980;40:365-369.

66. Stapleton RD. Biodegradation of aromatic hydrocarbons in an extremely acidic environment. Appl Environ Microbiol. 1998;64:4180-4184.

67. Staunton S. Effect of $\mathrm{pH}$ and some organic anions on the solubility of soil phospphate: implications for P bioavailability. Eur J Soil Sci. 1996;47: 231-239.

68. Singh BK, Walker A, Morgan JA, Wright DJ. Effects of soil $\mathrm{pH}$ on the biodegradation of chlorpyrifos and isolation of a chlorpyrifos-degrading bacterium. Appl Environ Microbiol. 2003;69:5198-51206.
69. Sharpley AN. Efect of soil pH on cation and anion solubility. Commun Soil Sci Plant Anal. 1991;22:1391-1399.

70. Reynolds ES. The use of lead citrate at high $\mathrm{pH}$ as an electron opaque strain in electron microscopy. J Cell Biol. 1963;17:208-212.

71. Baker KH. Bioremediation of surface and subsurface soils. New York: McGraw-Hill; 1994

72. Verstraete WR, Vanloocke R, De Borger R, Verlinde A. Modeling of the breackdown and the molbilization of hydrocarbons in unsaturated soil layers. En: Proceedings of the 3rd International Biodegradation Symposium.London Applied Science Publishers; 1976 .p. 98-112.

73. Haigler BE, Pettigrew CA, Sppain JC. Biodegradation of mixtures of substituted benzenes by Pseudomonas strain JS150. Appl Environ Microbiol. 1992;58:2237-2244.

74. Keel C, Weller DM, Natsch A, Defago G, Cook RJ. Thomashow LS. Conservation of the 2,4-diacetylphloroglucinol biosynthesislocus among fluorescent Pseudomonas strains from diverse geographic locations. Appl Environ Microbiol. 1996;62:552-563.

75. Kerry E. Microorganism colonizing plants and soil subjected to different degrees of human activity, including petroleum contamination in the Vestfold Hills and MacRobertson Land Antarctica. Polar Biol. 1990;10:423-430.

76. Pucci GN, Härtig C, Pucci OH. Influence of salinity and temperature on fatty acid composition of Pseudomonas fluorescens GNP-OHP-3 membrane. Rev Argent Microbiol. 2004;36:6-15. 